REVISTA 89.1

Revista RELACIONES INTERNACIONALES

Escuela de Relaciones Internacionales.

Universidad Nacional, Costa Rica.

N. ${ }^{\circ} 89.1$ • Enero-Junio de 2016

doi: http://dx.doi.org/10.15359/ri.89-1.6

Pp. 123-149

\title{
LA GESTIÓN DE LA INNOVACIÓN Y LA COMPETITIVIDAD DESDE LA POLÍTICA PÚBLICA EN COSTA RICA
}

\author{
Azucena Sandoval Barraza* \\ Juan Carlos Bermúdez Mora** \\ Jeannette Valverde Chaves ${ }^{* * *}$
}

\section{RESUMEN:}

La ciencia, la tecnología y la innovación desarrollada por las empresas son factores determinantes en el desarrollo socioeconómico de las regiones. Por ello el estudio aborda los mecanismos de gestión de la innovación en un sentido amplio, de acuerdo con la OECD (2011) desde el enfoque basado en los resultados, en el comportamiento y en los cambios de manera que constituye esta una herramienta muy valiosa para la competitividad de las empresas.

Palabras clave: gestión, innovación, competitividad, empresas, Costa Rica.

\section{ABSTRACT:}

Science, technology and innovation developed by enterprises, are crucial to the economic development of the regions factors. Therefore, the study analyzes the management mechanisms of innovation in a broad sense, according to the OECD (2011) from the results-based approach, behavior and changes so that it constitutes a valuable tool for the competitiveness of enterprises.

Keywords: Management, innovation, competitiveness, companies.

* Candidata Doctorado Ciencias Administrativas, Universidad de Occidente, Sinaloa, México. Labora como profesora Facultad de Ciencias Económicas, Universidad Autónoma de Sinaloa. Correo electrónico: azucena_sandoval@msn.com

** Ingeniero en Producción Industrial, Instituto Tecnológico de Costa Rica. Máster of Businness Administration, National University, CA., EE.UU. y Universidad de Costa Rica. Doctor en Ciencias Económicas y Empresariales, Universidad Latina de Costa Rica, con un posdoctorado en Economía de la Innovación, Universidad Autónoma de Sinaloa, México. Actualmente es catedrático de la Escuela de Relaciones Internacionales de la Universidad Nacional de Costa Rica. Correo electrónico: juan.bermúdez.mora@una.cr

*** Doctora en Estudios Latinoamericanos con énfasis en Pensamiento Latinoamericano, Máster en Relaciones Internacionales con énfasis en Negocios Internacionales, ambos por la Universidad Nacional de Costa Rica y especialidad en Responsabilidad social empresarial por la Universidad de Buenos Aires, Argentina. Es catedrática de la Universidad Nacional de Costa Rica. Labora como docente e investigadora en la Escuela de Relaciones Internacionales en la Universidad Nacional de Costa Rica. Correo electrónico: Jeannette. valverde.chaves@una.cr 


\section{Introducción}

El ambiente donde se desenvuelven las empresas ha sido estudiado por la teoría neoclásica de la economía en donde irrumpe el análisis shumpeteriano que permite el rompimiento de los ciclos, con la aparición de las innovaciones en el mercado, desencadenando cambios cualitativos y cuantitativos en el entorno económico (Brunet y Baltar, 2010); además, el papel de la empresa en cuanto a sus actividades internas tiene incidencia en las transformaciones del entorno.

En este sentido, Shumpeter enlaza aspectos microeconómicos de la teoría neoclásica con aspectos macroeconómicos de la escuela austriaca mediante el análisis mesoeconómico, en este último se desenvuelven las empresas que destruyen creativamente la estructura de mercado que relativamente prevalece en un momento y espacio histórico.

El artículo sigue el enfoque basado en el comportamiento, por considerar la relación de las nuevas formas de innovación con procesos para organizar el proceso innovativo; define innovación, gestión de la innovación y plantea las acepciones de Michael Porter para el estudio de la competitividad.

\section{Concepto y clasificación de mecanismos de gestión de la innovación}

Para llegar a la definición de mecanismos de gestión de la innovación, se consideró necesario fragmentar el concepto por definición de mecanismos, gestión de la innovación e innovación, después las representaciones de la cadena de valor y al final se reconstruye "mecanismos de gestión de la innovación", para el sentido del presente documento.

\section{a. Definición de mecanismos, innovación y gestión de la innovación}

El origen de las palabras mecanismos, gestión de la innovación e innovación nos proporcionará una perspectiva sobre el uso actual de ellos, se pretende dar coherencia y significado desde lo particular a lo general. Se presentan las siguientes definiciones:

Mecanismo: La palabra se deriva del latín mechanisma, de los vocablos griegos mechane (instrumento para levantar objetos) y mechos (medios para hacer algo o modo de hacer algo), en español incluye la definición de conjunto de partes de una máquina en su disposición adecuada; estructura de un cuerpo natural 
o artificial, y combinación de sus partes constitutivas, medios prácticos empleados en las artes y, proceso, según la Real Academia de la Lengua Española; sin embargo, en The New Palgrave: A Dictionary of Economics (2008) encontramos que mecanismo es una descripción de cómo los individuos toman decisiones económicas en función de la información que poseen, en este documento es el proceso que llevan a cabo las empresas en cuanto a decisiones económicas administrativas en función de la información que posee la unidad económica.

Innovación: Para Shumpeter, innovación es el resultado de la investigación y desarrollo invertidos en un nuevo producto o proceso que llega al mercado (Manual de Oslo, 2005). En el Diccionario LID innovación (2010) se define la innovación como creatividad implantada con éxito de tal forma que produce un valor añadido, la cual puede aparecer en productos, servicios, modelos de negocio, estrategias, entre otros; además se presenta en forma abierta, cerrada, disruptiva o incremental. En el Manual de Oslo la innovación es la introducción de un nuevo o significativamente mejorado producto (bien o servicio), proceso, nuevo método de comercialización u organizativo en las prácticas internas de la empresa, organización del trabajo o relaciones exteriores; en el Manual de Bogotá (2001) se incorpora el esfuerzo tecnológico a la definición.

Gestión de la innovación: De acuerdo con el Manual de Bogotá se desplaza el eje de análisis sobre innovación y queda como esfuerzo tecnológico o gestión de actividades innovadoras; la justificación reside en que en las regiones de países latinoamericanos las empresas no alcanzan un fuerte nivel de tecnificación que se remita a la estricta definición de innovación del Manual de Oslo. Para Álvarez y Botella (2012), los países de América Latina requieren diferentes combinaciones de instrumentos, por ello se incorpora el esfuerzo tecnológico como investigación y desarrollo, diseño, instalación de maquinarias nuevas, modernización de la organización, comercialización y capacitación (Manual de Bogotá, 2001). Por otra parte, el Manual de COTEC realiza una definición de la gestión de la innovación tecnológica como la organización y dirección de los recursos humanos y económicos, con el fin de aumentar la creación de nuevos conocimientos, generación de ideas técnicas que permitan obtener nuevos productos, procesos y servicios, o bien, mejorar los ya existentes (Fundación Cotec, 2001).

Con base en las anteriores definiciones, en esta investigación se le llama mecanismos de gestión de la innovación de las empresas al proceso que decide la empresa seguir en cuanto a la administración de los recursos humanos y económicos, para crear nuevos conocimientos que permitan obtener innovaciones a través de la investigación y desarrollo, diseño, adquisición de tecnología, modernización organizacional, comercialización y capacitación. 
El argumento del uso de la anterior definición se basa en que Sinaloa es una región latinoamericana donde las empresas analizadas no presentan alto nivel de desarrollo tecnológico en comparación con países desarrollados, por lo tanto, la conceptualización mayormente apegada al Manual de Bogotá se considera adecuada. A continuación se presentan los tipos de innovación.

\section{Tipos de innovación}

De acuerdo con el Diccionario LID sobre innovación (2010), este concepto tiene varias acepciones, entre las que se destacan las siguientes:

- Innovación de proceso. Creación y utilización de procesos novedosos como en venta, gestión, producción, operaciones, entre otros.

- Innovación de producto. Proceso de creación y de lanzamiento de un producto nuevo, se incluye la gestión y el resultado del proceso.

- Innovación de servicio. Proceso de creación y de lanzamiento de un servicio nuevo, se incluye la gestión y el resultado del proceso.

- Innovación no tecnológica (organización). Tipo de innovación que abarca aquellos ámbitos de la empresa que suponen cambios sustanciales en su comportamiento estratégico y en sus resultados, y que no están ligados directamente al concepto tradicional de innovación de producto o de proceso.

- Innovación de mercado. Proceso de descubrimiento y de aprovechamiento de una nueva estructura de mercado, así como el resultado del dicho proceso.

- Innovación en modelo de negocio. Proceso de desarrollo y de gestión de cambios sistémicos de un modelo de negocio para crear un nuevo modelo de negocio o para mejorar un modelo de negocio ya existente.

- Innovación estratégica. Tipo de innovación transformadora que puede incluir innovaciones en proceso, en producto o en servicio, pero donde ya existe un nuevo modelo de negocio con alto potencial de crecimiento.

- Innovación accidental por fenómeno natural. Innovación que se produce por aprovechamiento directo de un fenómeno natural. 
- Innovación accidental por implantación de fenómeno. Innovación que se produce por una adaptación de un fenómeno natural a un entorno determinado. Implica cierta comprensión del fenómeno.

- Innovación agrícola. Desarrollo e implantación de una idea nueva en el ámbito de la agricultura.

- Innovación accidental por observación y modificación de fenómeno. Innovación que se produce al comprender profundamente y al modificar un fenómeno natural.

- Innovación bajo demanda. Situación en la que la necesidad de cambio o en la que un cambio en las condiciones ambientales hace imprescindible encontrar una nueva solución.

- Innovación basada en circunstancias climatológicas. Corriente de pensamiento en relación con las teorías geográficas para explicar la historia de la innovación.

- Innovación como copia. Situación en la que la estrategia de innovación pasa por copiar a los competidores de una manera muy rápida y eficiente.

- Innovación como estrategia. Situación en la que las condiciones ambientales demandan la producción constante de nuevas soluciones.

- Innovación con cliente. Cada uno de los procesos de innovación en los que el usuario del producto o del servicio es partícipe. Este término fue acuñado por Eric Von Hipil y es un caso particular de innovación abierta.

- Innovación con pensamiento evolutivo. Técnica desarrollada en los años 60 por syntectics para desarrollar conceptos nuevos mediante una respuesta por ítems hasta convertirlos en algo que aporte valor en su implantación.

- Innovación ensayo y error. Primer método para innovar conocido que se basa en la realización de experimentos; descarta aquellos que no tengan los resultados deseados.

- Innovación inspirada en el mundo animal. Técnica basada en la observación del mundo animal para diseñar innovaciones.

- Innovación mejora continua. Técnica desarrollada en los años 1960 en la que se van incorporando pequeñas mejoras contantemente con el objetivo de optimizar una determinada operación. 
- Innovación método científico. Situación en la que los vectores de inercia psicológica están basados en un conocimiento profundo del mundo natural $\mathrm{y}$ de todos los experimentos realizados previamente en dicha área

- Innovación oportunista. Técnica mediante la cual se observa un fenómeno de forma accidental para reproducirlo de manera sistemática.

- Innovación por aprovechamiento de efecto científico. Técnica mediante la cual se usa el descubrimiento científico y se le dota de una aplicación práctica.

- Innovación sistemática. Metodología abierta de innovación con origen en el conjunto de conceptos y de técnicas de TRIZ, tiene por objeto sistematizar el proceso de innovación y resolución inventiva en problemas.

- Innovación social. Desarrollo e implantación de una idea nueva en el ámbito social.

- Innovación tecnológica. Actividad cuyo resultado supone un avance tecnológico en la obtención de nuevos productos, procesos de producción o mejoras sustanciales tecnológicamente significativas

El citado Diccionario de innovación (2010) señala que estos tipos de innovación se presentan mediante las siguientes formas:

- Innovación abierta. Estrategia de innovación en la que elementos externos a la organización desempeñan un papel fundamental en el desarrollo de nuevos productos, servicios y procesos.

- Innovación cerrada. Estrategia de innovación de las organizaciones donde la innovación se gestiona exclusivamente a través de medios y conocimientos internos. Actualmente la gran mayoría de las organizaciones la usan.

- Innovación incremental o significativa. Añade mejoras a lo existente o recombina de una manera distinta, de manera que resulta en algo nuevo, con un grado de novedad relativamente baja. Se presenta en cualquier industria o actividad, va a depender de las presiones de demandas, aspectos sociales y culturales, y de las oportunidades y trayectorias tecnológicas de la empresa (Freeman, 2008).

- Innovación radical, transformacional, discontinua o disruptiva. Representa algo totalmente nuevo en el mundo, aporta cambios radicales en 
lo conocido. Son eventos discontinuos que resultan de la investigación y desarrollado realizados por empresas, universidades o gobierno, según Freeman (2008).

Planteadas las diferentes definiciones de innovación, se conceptualiza la competencia, estrategia competitiva y ventaja competitiva por considerarse la innovación un elemento necesario para llevar a cabo los conceptos mencionados y como parte de la cadena de valor.

\section{Definición de competencia, estrategia competitiva y ventaja competitiva}

Se define en este apartado lo que es competencia, estrategia competitiva y ventaja competitiva para entender la esquematización de la cadena de valor retomada de Michael Porter, enfocándose en las actividades de innovación como el desarrollo tecnológico.

Competencia: De acuerdo a The palgrave enciclopedia of strategic management (2013) la competencia consiste en determinar las estructuras y los procesos por parte de las empresas para establecer los precios del mercado y la asignación de recursos. Existen tres tipos: competencia para los estrategas, la competencia estática y la teoría de juegos.

Estrategia competitiva: The palgrave enciclopedia of strategic management la define como las acciones que las empresas llevan a cabo -y los resultados de rendimiento asociados con esas acciones-, que reconocen la existencia de competidores directos y de otras empresas que compiten por los beneficios.

Ventaja competitiva: La ventaja competitiva es el grado en el que una empresa crea más valor económico que las empresas rivales en un mercado de producto dado, según The palgrave enciclopedia of strategic management.

\section{Herramientas para el análisis de la gestión de la innovación}

Las herramientas utilizadas para el estudio de los mecanismos de gestión de la innovación se centran en tres: Manual de Bogotá, Manual de COTEC y Cadena de valor de Michael Porter. Estos permiten estudiar la conducta de las regiones, en particular la de las empresas y su interacción con la innovación, como a continuación se describe. 


\subsection{Manual de Bogotá}

El Manual Latinoamericano de Indicadores de Innovación Tecnológica, mejor conocido como Manual de Bogotá, surge de la necesidad de establecer indicadores de procesos de innovación en América Latina; independientemente de la diferencia en dimensiones geográficas de los países, existen cuestiones sociológicas, económicas, culturales y políticas que son características de una región, por lo cual se debe analizar desde esa perspectiva para lograr reflejar la realidad del fenómeno; pero, al mismo tiempo y a pesar de las diferencias, se cuente con indicadores que permitan hacer comparaciones con procesos de innovación de diferentes partes del mundo.

Cabe mencionar el aspecto de la medición: en manuales anteriores, como el de Oslo y Frascati, se enfocaba el análisis en aspectos cuantitativos; el manual de Bogotá recupera la experiencia del Manual de Oslo e incorpora aspectos cualitativos, con la finalidad de capturar la trayectoria de la economía con base en aspectos de innovación, para formular políticas públicas adecuadas para las características de cada región, pues las regiones latinoamericanas no cuentan con suficiente desarrollo como las de economías desarrolladas. Por tanto, la región estudiada en la investigación se adapta a las conceptos del Manual de Bogotá en el sentido de considerar el esfuerzo tecnológico o la gestión de la actividad innovadora (Manual de Oslo, 2005), a la que se denomina gestión de la innovación.

Los mecanismos de gestión de la innovación que llevan a cabo las empresas son de mayor relevancia por encontrarse inmersas en transformaciones más veloces en cuanto al impulso de innovación y competitividad a través de la ciencia y la tecnología. Menciona León (2008) que los gobiernos y organismos internacionales consideran crucial la diseminación y el uso del conocimiento, el cual, dadas sus contribuciones potenciales, ha adquirido un nivel de significación a través de la creación de la riqueza, la elevación de la calidad de vida y el mejoramiento de las políticas públicas, justificando, así, los recursos financieros destinados a la causa. Es en las organizaciones de soporte a la innovación donde germina la inteligencia, considerada elemento clave para el desarrollo de las regiones.

Los elementos que se retoman para el estudio de los mecanismos de gestión de la innovación del Manual de Bogotá (2001) son las innovaciones y las actividades de innovación. 


\section{Innovación}

Las innovaciones pueden ser de productos, procesos, organización y comercialización; son implementaciones tecnológicas nuevas, o bien, mejoras tecnológicas; respecto a la organización, deben ser cambios en las formas de organización y administración del proceso; las de comercialización corresponden a nuevos métodos de entregar el producto obtenido, así como nuevas formas de mercadeo.

\section{Actividades de innovación}

Son las actividades que realiza la empresa para lograr obtener nuevos conocimientos; estas son investigación y desarrollo, y esfuerzos de innovación, las cuales pueden producir un cambio técnico en la empresa sin llegar a la innovación tecnológica, estrictamente (Manual de Bogotá, 2001).

\section{- Investigación y desarrollo}

La investigación y desarrollo es el cúmulo de conocimientos a través del desarrollo de productos nuevos, procesos nuevos, prototipos y plantas pilotos; la investigación puede ser desde básica, estratégica, experimental o aplicada (Manual de Bogotá, 2001).

\section{- Esfuerzos de innovación}

Los esfuerzos de innovación corresponden a acciones tendientes a acumular conocimiento. Son: diseño, adquisición de tecnología, comercialización y capacitación. Se consideran el capital físico, el capital humano y el capital de conocimiento (Manual de Bogotá, 2001).

\section{- Diseño, instalación de maquinarias nuevas, ingeniería industrial, puesta en marcha de la producción}

Se refiere a planos y gráficos específicos necesarios para introducir innovaciones. Es una actividad de apoyo que se puede realizar desde el diseño de productos hasta el de procesos. Además, contempla la adquisición de edificios o maquinarias, estas últimas pueden ser herramientas y equipo en general, con la característica de que las adquisiciones no mejoran el desempeño tecnológico; sin embargo, son útiles para la implementación de las innovaciones (Manual de Bogotá, 2001). 


\section{- Adquisición de tecnología incorporada al capital}

Es toda la maquinaria y equipo que adquiere la empresa para el mejoramiento del desempeño tecnológico, inclusive el software; contempla la maquinaria y equipo vinculados con las innovaciones ya implementadas por la empresa (Bogotá, 2001).

\section{- Adquisición de tecnología no incorporada al capital}

Son las patentes, licencias, marcas, software, inventos, patrones y demás servicios científicos vinculados a las innovaciones de producto y proceso (Manual de Bogotá, 2001).

\section{- Modernización organizacional}

Son cambios en la organización de la empresa para mejorar la eficiencia, como tiempo empleado, calidad en servicio, en producto, mejora en líneas de producción, entre otros. Estos conducen al cambio en la organización del proceso productivo, por ejemplo, en la línea de producción, en la organización física de la planta, outsourcing, entre otros (Manual de Bogotá, 2001).

\section{- Comercialización}

Actividades relacionadas con la comercialización del producto o servicio que se desea colocar en el mercado, como investigación de mercado, publicidad de los productos o servicios, mejora en la penetración de mercados específicos mediante cambios en la presentación o entrega del producto (Manual de Bogotá, 2001).

\section{- Capacitación}

Es una capacitación en aspectos de innovación tecnológica, es decir, con el desarrollo de productos, procesos, organización, manejo de maquinaria especializada, entre otros. El manejo de tecnologías ya sea blandas (gestión y administración) o duras (tecnología de procesos productivos) requiere de personal capacitado en estos temas (Manual de Bogotá, 2001).

\subsection{Manual de COTEC}

El manual de la Fundación para la Innovación Tecnológica (COTEC) presenta la gestión de la innovación como la organización y dirección de los recursos, 
tanto humanos como económicos, con el fin de aumentar la creación de nuevos conocimientos; la generación de ideas técnicas que permitan obtener nuevos productos, procesos y servicios o mejorar los ya existentes; el desarrollo de dichas ideas en prototipos de trabajo, y la transferencia de esas mismas ideas a las fases de fabricación, distribución y uso, en respuesta a las necesidades del cliente y del mercado. Se trata de un concepto que abarca a todas las áreas funcionales y servicios de la empresa, basado en el modelo de gestión que predica la metodología TEMAGUIDE de Cotec. Esta herramienta fue analizada para acumular el acervo intelectual; sin embargo, después de revisar los estudios hechos por COTEC a empresas sobre todo del continente europeo, se ha descartado ponerla en práctica en las empresas de la región de Sinaloa. Cabe mencionar que los conceptos y metodología de COTEC sirvieron como directrices y de apoyo académico para el estudio de campo y los resultados encontrados.

El manual distingue dos tipos de procesos: los nucleares y los facilitadores. Los procesos nucleares son aquellos directamente involucrados en la innovación tecnológica, cuyos resultados están relacionados con la transformación de oportunidades e ideas en innovaciones con un impacto en el mercado. Los procesos y prácticas facilitadores no son exclusivos de la gestión de la innovación tecnológica, pero sirven de apoyo a los procesos nucleares; son igualmente necesarios para que los procesos nucleares puedan funcionar de manera efectiva (Cotec, 2008).

\section{Procesos nucleares}

Los procesos nucleares son considerados los que están directamente vinculados con la innovación tecnológica; estos son la estrategia tecnológica, la adquisición de tecnología, el desarrollo de productos o servicios y la innovación de procesos.

\section{- Estrategia tecnológica}

Principalmente es representada por un plan tecnológico, el cual es la guía que sigue la empresa con objetivos bien planteados hacia la innovación.

\section{- Desarrollo de productos o servicios}

Todas las actividades que conduzcan a la elaboración de un producto o un servicio que puede ser introducido en el mercado. 


\section{- Innovación de procesos de contenido tecnológico}

Todas las actividades que conduzcan al logro de un nuevo proceso hasta que sea puesto en práctica con éxito por la empresa.

\section{- Adquisición de tecnología}

La compra de tecnología externa que puede ser tangible, así como el desarrollo de intangibles tecnológicos al interior de la empresa, el cual es denominado investigación.

\section{Procesos y prácticas facilitadoras}

Son las actividades que sirven de apoyo para que se logren las innovaciones. Son la organización de investigación y desarrollo tecnológico, gestión de recursos humanos, colaboración con terceros, gestión y aseguramiento de la calidad, vigilancia tecnológica, y gestión de proyectos.

\section{- Organización de investigación y desarrollo tecnológico}

Identificación de la forma que usa la empresa para emprender actividades de innovación, esto incluye el financiamiento, la incorporación de tecnología externa e interna, entre otros.

\section{- Recursos humanos}

La gestión de recursos humanos capacitados para desempeñarse en actividades de investigación y desarrollo tecnológico.

\section{- Colaboración con terceros}

Relaciones que se tienen con agentes del exterior como proveedores, clientes, universidades, gobierno, entre otros.

\section{- Gestión y aseguramiento de la calidad}

Medidas que generan pautas a seguir para cumplir con requisitos de calidad. 


\section{- Vigilancia tecnológica}

Información del entorno, comúnmente es sobre la competencia y el comportamiento del mercado para elegir la estrategia correcta.

\section{- Gestión de proyectos}

En ocasiones puede ser la estrategia que la empresa adopta, ya que la gestión de proyectos afecta a toda la organización porque está directamente relacionada con las tareas de innovación.

De acuerdo con lo anterior, la empresa debe desarrollar su modelo de gestión de la innovación tecnológica relacionado con el sector donde se desempeña; su magnitud en cuanto a tamaño y la estrategia por la que haya decidido optar se enuncia literalmente en la relación que guarda la innovación con la empresa y el sistema de innovación (Fundación Cotec, 2010, p. 14):

La potenciación de la capacidad de innovación pasa por una mayor colaboración tanto con organizaciones de investigación como dentro de la cadena de valor del negocio y por una mejor gestión de la misma, a través de instrumentos como los marcos estables de colaboración. Todas las empresas que buscan el liderazgo necesitan contar con una cultura innovadora que haga eficaz un sistema de innovación bien definido y con una cultura innovadora que haga eficaz un sistema de innovación bien definido y estructurado. De esta manera, la organización funciona "sin costuras" y la innovación se produce de manera natural.

\subsection{Cadena de valor}

La cadena de valor es una herramienta presentada por Michael Porter para el estudio de las ventajas competitivas de las empresas en determinadas industrias, pero antes se considera importante señalar el antecedente de la competencia, considerada un beneficio para las personas y organizaciones de cualquier tipo de área o asociación. La naturaleza de la competencia, esta es vital para cualquier sistema económico dinámico. En realidad no puede ser de otra manera, ya que en sociedades estáticas, la competencia se da de forma natural y desde Darwin existe la competencia por adaptarse a los constantes cambios, se está compitiendo para poder sobrevivir.

En el ambiente empresarial, la empresa busca superar a sus competidores a través de una actuación dirigida a ganar en el mercado; la toma de decisiones y la elección de alternativas diferentes a su competencia es la clave para vencer. 
Para Porter (2005), la verdadera competitividad se mide por la naturaleza y productividad de las actividades económicas que se desarrollan, aquí es donde entra la estrategia competitiva porque examina esas acciones que llevan a cabo las empresas.

\subsubsection{Cinco fuerzas competitivas}

Para diseñar la estrategia competitiva, la empresa debe conocer sus condiciones, sus competidores, sus objetivos, las políticas que la rigen y cómo está relacionada con su ambiente. Dentro de su ambiente es imperante conocer la estructura económica en la que se suscribe, la cual dependerá de las cinco fuerzas competitivas (ver figura 1): 


\section{Participantes potenciales}

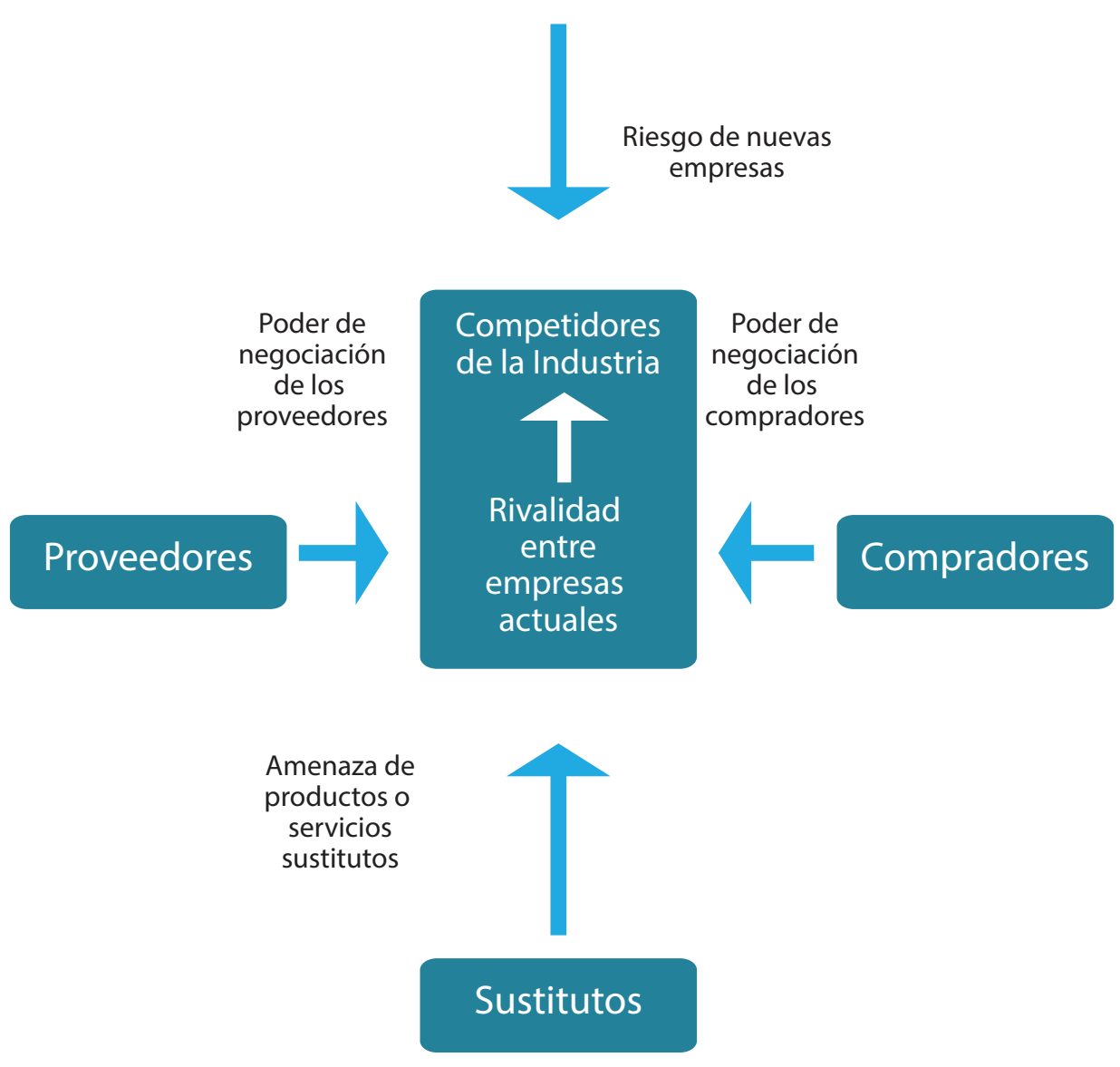

Figura 1. Fuerzas competitivas de Michael Porter (2002). 
Las cinco fuerzas competitivas son dinámicas y, al unirse, muestran la intensidad de la competencia en un sector; cada una de ellas requiere de un análisis profundo que, en esta ocasión, se remitirá a su mención:

En el riesgo de entrada a más participantes, se encuentren las barreras contra la entrada, las principales fuentes son:

1. Economías de escala

2. Diferenciación de productos

3. Necesidades de capital

4. Costos cambiantes

5. Acceso a los canales de distribución

6. Desventajas de costos independientes de las economías de escala:

a. Tecnología de productos patentados

b. Acceso preferencial a materias primas

c. Ubicación favorable

d. Subsidios gubernamentales

e. Curva de aprendizaje o de experiencia

f. Política gubernamental

En la intensidad de la rivalidad de competidores actuales se encuentran factores estructurales como:

1. Competidores numerosos o de igual fuerza

2. Lento crecimiento de la industria

3. Altos costos fijos o de almacenamientos 
4. Ausencia de diferenciación o costos cambiantes

5. Aumento de la capacidad en grandes incrementos

6. Competidores diversos

7. Importantes intereses estratégicos

8. Barreras sólidas contra la salida:

a. Activos especializados

b. Costos fijos de la salida

c. Interrelaciones estratégicas

d. Barreras emocionales

e. Restricciones gubernamentales y sociales

9. Rivalidad cambiante

10. Barreras contra la salida y la entrada

La presión que ejercen los productos sustitutos limita los rendimientos potenciales de la industria; de manera general, estos pueden ser los que mejoran la relación de precio-desempeño con el actual producto de la industria y aquellos que generan altas utilidades.

Con respecto al poder de los compradores, se considera importante si cumplen con lo siguiente:

1. El grupo está concentrado o compra grandes volúmenes en relación con las ventas del proveedor.

2. Los productos que compra el grupo a la industria representan una parte considerable de los costos o de las adquisiciones que realiza.

3. Los productos que el grupo adquiere en la industria son estándares o indiferenciados. 
4. El grupo tiene pocos costos cambiantes.

5. El grupo obtiene baja utilidades.

6. Los compradores representan una seria amenaza contra la integración hacia atrás.

7. El producto de la industria no es decisivo para la calidad de los productos del grupo ni para sus servicios.

8. El grupo tiene toda la información.

En cuanto a los proveedores, se considera importante si cumplen lo siguiente:

1. El grupo está dominado por pocas compañías y muestra mayor concentración que la industria a la que le vende.

2. El grupo de proveedores no está obligado a competir con otros productos sustitutos para venderle a la industria.

3. La industria no es un cliente importante para el grupo de proveedores.

4. El grupo de los proveedores es un insumo importante para el negocio del comprador.

5. Los productos del grupo de proveedores están diferenciados o han acumulado costos cambiantes.

6. El grupo de proveedores constituye una amenaza seria contra la integración vertical.

\subsubsection{Estrategia competitiva}

La estrategia competitiva son las acciones que, en lo particular, la empresa decide tomar para defenderse en una industria competitiva, frente a las cinco fuerzas competitivas. Son tres las estrategias genéricas de las que se puede disponer para tener un desempeño mejor que el de los rivales de la industria: 
1. Liderazgo global en costos

\section{Diferenciación}

3. Enfoque o concentración

Tal y como se muestra en la siguiente tabla 1, el autor Michael Porter (1991) plantea los siguientes recursos, habilidades y necesidades de la organización para las estrategias genéricas.

Tabla 1.

Recursos, habilidades y necesidades de la estrategia

\begin{tabular}{|c|c|c|}
\hline Estrategia genérica & $\begin{array}{l}\text { Habilidades y recursos } \\
\text { requeridos comúnmente }\end{array}$ & $\begin{array}{l}\text { Necesidades } \\
\text { organizacionales comunes }\end{array}$ \\
\hline $\begin{array}{l}\text { Liderazgo en costos } \\
\text { globales }\end{array}$ & $\begin{array}{l}\text { Inversión sostenida de capital y } \\
\text { acceso a los capitales } \\
\text { Habilidades de ingeniería de } \\
\text { procesos } \\
\text { Supervisión meticulosa de la } \\
\text { mano de obra } \\
\text { Productos diseñados } \\
\text { para facilitar la manufactura } \\
\text { Sistema barato de distribución }\end{array}$ & $\begin{array}{l}\text { Riguroso control de costos } \\
\text { Informes detallados } \\
\text { y frecuentes de control } \\
\text { Organización y } \\
\text { responsabilidades bien } \\
\text { estructuradas } \\
\text { Incentivos basados en el } \\
\text { cumplimiento de objetivos } \\
\text { cuantitativos estrictos }\end{array}$ \\
\hline
\end{tabular}




\begin{tabular}{|l|l|l|}
\hline Diferenciación & $\begin{array}{l}\text { Sólidas capacidades de mar- } \\
\text { keting }\end{array}$ & $\begin{array}{l}\text { Buena coordinación entre las } \\
\text { funciones de investigación y } \\
\text { desarrollo, de desarrollo de } \\
\text { productos y de marketing }\end{array}$ \\
& $\begin{array}{l}\text { Engeniería de productos } \\
\text { Gran capacidad de investiga- } \\
\text { ción básica }\end{array}$ & $\begin{array}{l}\text { Medición subjetiva e in- } \\
\text { centivos en vez de medidas } \\
\text { cuantitativas }\end{array}$ \\
& $\begin{array}{l}\text { Reputación corporativa del } \\
\text { liderazgo tecnológico o en } \\
\text { calidad }\end{array}$ & $\begin{array}{l}\text { Comodidades para atraer } \\
\text { mano de obra muy bien cali- } \\
\text { ficada, científicos o personas } \\
\text { creativas }\end{array}$ \\
& $\begin{array}{l}\text { Larga tradición en la industria } \\
\text { o combinación original de } \\
\text { habilidades obtenidas de otras } \\
\text { industrias }\end{array}$ & $\begin{array}{l}\text { Cooperación incondicional de } \\
\text { otros canales }\end{array}$ \\
$\begin{array}{l}\text { Combinación de las políticas } \\
\text { anteriores dirigidas a determi- } \\
\text { nado objetivo estratégico }\end{array}$ & $\begin{array}{l}\text { Combinación de las políticas } \\
\text { anteriores dirigidas a deter- } \\
\text { minado objetivo estratégico }\end{array}$ \\
\hline Enfoque & & \\
\hline
\end{tabular}

Nota: Porter (1991).

\subsubsection{Actividades de valor}

Una forma de examinar las actividades que desempeña la industria y, en lo particular, la estrategia de diferenciación de la empresa, es mediante el análisis de la ventaja competitiva a través de la herramienta la cadena de valor; como ya se mencionó anteriormente en esta investigación, fue de gran utilidad utilizarla para poder identificar las actividades que le dan valor a la empresa. De acuerdo con Porter (1991), se pueden dividir en actividades primarias y actividades de apoyo, las primeras implican la creación física del producto, su venta, transferencia y asesoría posterior a la venta; las segundas son apoyo de las primeras, proporcionan insumos, tecnología, recursos humanos, entre otros. 
Actividades primarias:

1. Logística interna

2. Operaciones

3. Logística externa

4. Mercadotecnia y ventas

5. Servicio

Actividades de apoyo:

1. Abastecimiento

2. Desarrollo de tecnología

3. Administración de recursos humanos

4. Infraestructura de la empresa

\section{$5 \quad$ Gestión de la innovación y la competitividad en Costa Rica}

La competitividad, concebida como la capacidad que tiene una empresa, organización o país de obtener rentabilidad en el mercado en relación con sus competidores, depende de la relación entre el valor y la cantidad del producto ofrecido y los insumos necesarios para obtenerlo (productividad), y la productividad de los otros oferentes del mercado.

Por lo anterior, en Costa Rica, desde el año 2006, se creó mediante Decreto 33150 del 9 de mayo del 2006, el Consejo Nacional de Competitividad que tiene a su cargo entre otras funciones:

1T) Proponer y presentar para aprobación del Consejo de Gobierno del Poder Ejecutivo el Plan Nacional de Competitividad (PNC). T T2) Diseñar y presentar para aprobación del Poder Ejecutivo los instrumentos de políticas de competitividad que sean necesarios para cumplir con los objetivos 
y metas estratégicas del Plan Nacional de Competitividad. T T3) Desarrollar y asegurar una efectiva ejecución del Plan Nacional de Competitividad como de los Planes Estratégicos de Acción y los Planes Operativos Anuales que lo componen. T T4) Realizar los análisis, estudios y diagnósticos estratégicos que permitan identificar las limitantes a la competitividad internacional del país, los sectores productivos y las regiones y su potencial... (CONACOM, 2006, p. 6)

Dicho Consejo concibe la competitividad como un sistema que involucra a todos los actores nacionales, para ello creó la Agenda Nacional de Competitividad 2016-2016, con una visión amplia que requiere la acción de múltiples actores. Esta opera en el contexto de una base territorial claramente definida. Su propósito ha sido identificar y promover los factores que originan la competitividad, por considerar que estos se encuentran entretejidos a nivel social, económico y cultural, y vinculados a ciertos contextos y lugares (CONACOM, 2006).

La Agenda Nacional de Competitividad, Costa Rica 2006-2016, busca apuntalar los siguientes seis ejes de acción estratégicos, tal y como se muestra en la siguiente figura 2: Sociedad educada y preparada al trabajo; 2 . Fomento y fortalecimiento institucional; 3. Desarrollo de infraestructura productiva y tecnológica; 4. Fortalecimiento del aparato productivo y exportador; 5. Desarrollo económico local; 6. Balance y sostenibilidad ambiental.

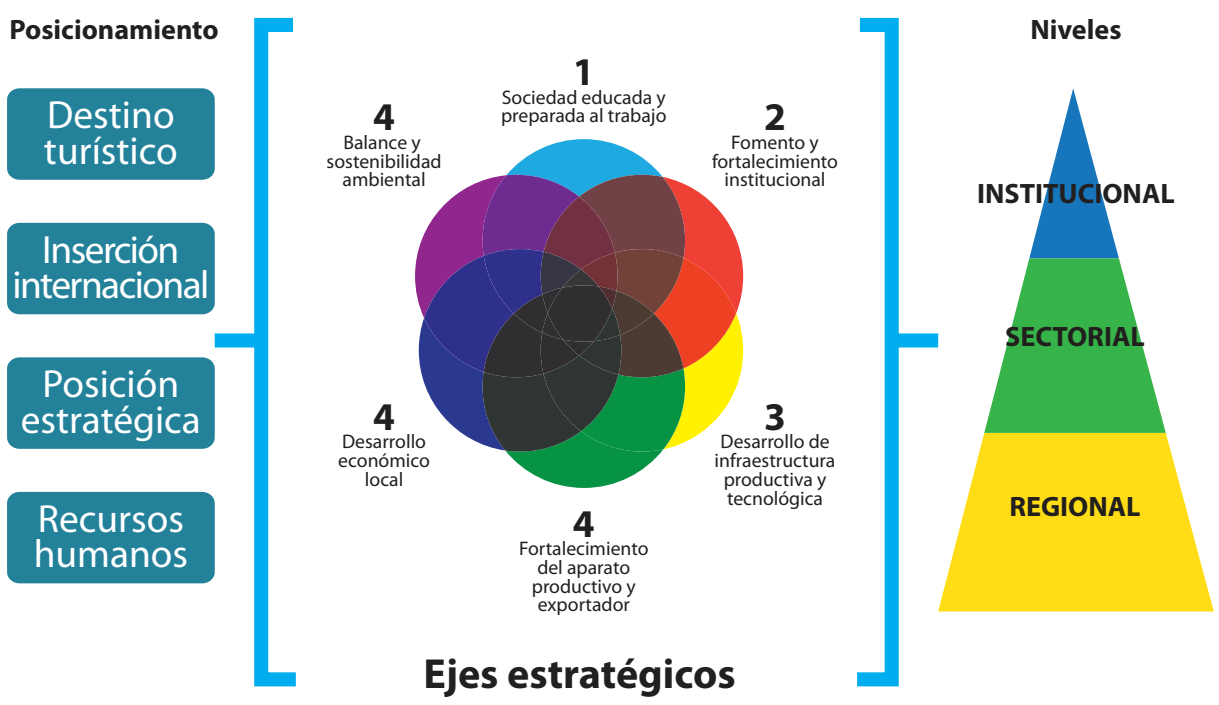

Figura 2. Agenda Nacional de Competitividad de Costa Rica 2006-2016. (CONACOM, 2006, p. 11). 
Dicha agenda ha ido avanzando según vaivenes políticos, aprovechando no solo la ubicación geográfica del país, sino las alianzas y vínculos internacionales para atraer inversiones, fomentar la actividad comercial internacional, creando capacidades en la población para que pueda enfrentar los retos y desafíos de la actual coyuntura con herramientas sólidas.

Tal y como se plantea en el Plan Nacional de Costa Rica 20014-2018, "el modelo de desarrollo económico de Costa Rica se ha basado durante las tres últimas décadas en el aumento y diversificación de las exportaciones, el fomento del turismo, el desarrollo de la alta tecnología y los servicios, así como el incremento en los flujos de inversión extranjera directa en el marco de un esquema de apertura comercial y libre comercio" (MIDEPLAN, 2014, p. 53).

Siguiendo con esa línea de trabajo, desde la política pública en Costa Rica se ha entendido que debe existir una estrecha relación entre el bienestar de las personas y el crecimiento económico ambientalmente sostenible, tal y como se muestra en la figura 3 .

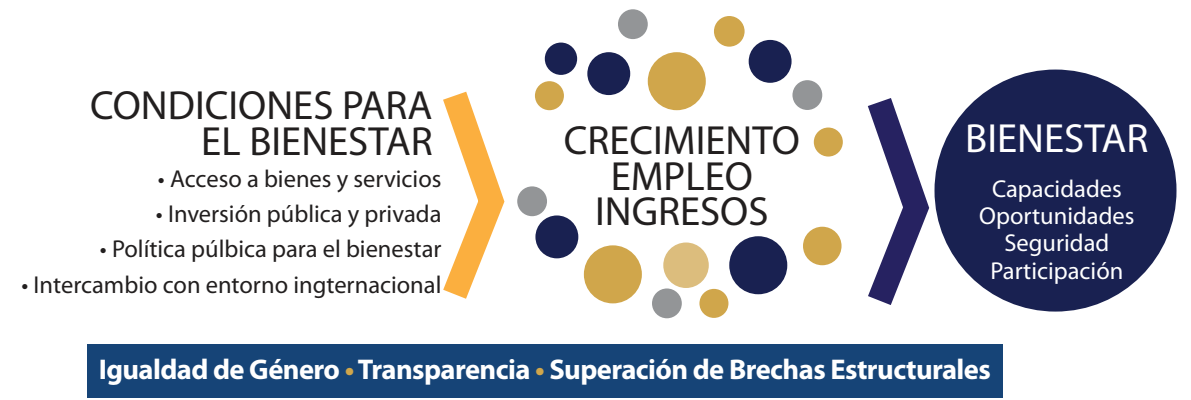

Figura 3. Relación entre el bienestar de las personas y el crecimiento económico ambientalmente sostenible. (Plan Nacional de Desarrollo, 2014, p. 30).

Desde la gestión de la política pública se plantea que "el Estado no solo provee bienes públicos y regula, sino que también puede animar la infraestructura económica y social que facilite el crecimiento económico y mejorar tanto la distribución de recursos como la productividad laboral mediante los gastos en salud, educación, energía, telecomunicaciones, ciencia y tecnología" (MIDEPLAN, 2014, p. 48).

En la figura 4 se observan algunas de las condiciones necesarias para dinamizar la economía y mejorar los índices de competitividad. 


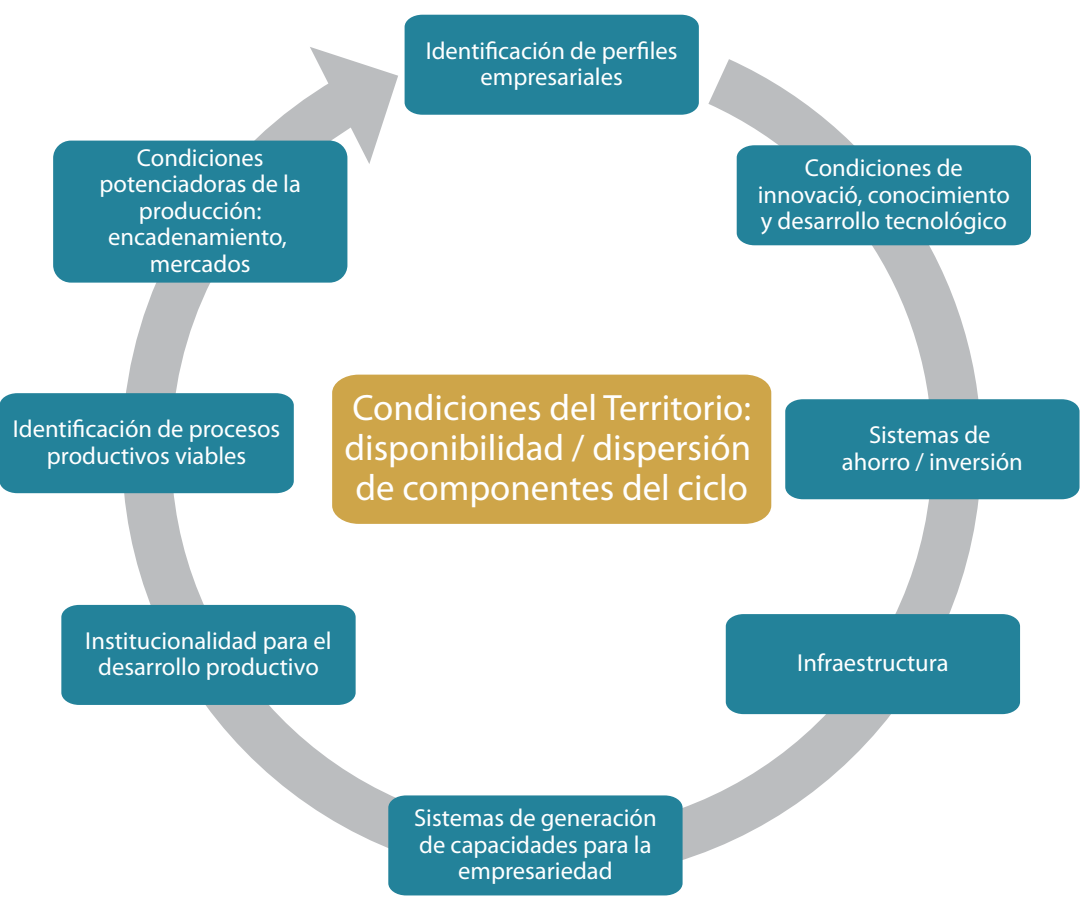

Figura 4. Condiciones para la dinamización económica.

(MIDEPLAN, 2014, p. 69).

Sin duda alguna, como se indica en el Informe Mundial de Competitividad (2014), el entorno institucional está determinado por el marco legal y administrativo en el que los individuos, empresas y gobiernos interactúan para generar riqueza. Dicho entorno institucional debe ser sólido y justo para que garantice la apertura y participación de todos los sectores productivos. En él, el Estado desempeña una función determinante para atestiguar calidad en las instituciones y para crear políticas que no solo fomenten la competitividad y la innovación sino que, además, garanticen la protección de los productos derivados de esa innovación.

\section{Conclusiones}

Tal y como lo señala el CONACOM, el clima de negocios y la sostenibilidad del crecimiento deben trabajarse tanto en el nivel sectorial, como el regional y el institucional, para causar los impactos deseados, ya que el énfasis del trabajo debe considerar las necesidades y propuestas de cada instancia. 
El crecimiento económico se fortalece en tanto se atiendan retos que inciden en la competitividad de los sectores productivos, relacionados con la infraestructura de transportes, costos de energía, simplificación de trámites, la disponibilidad de mano de obra capacitada, desarrollo micro empresarial, estabilidad macroeconómica y consolidación de mercados para el intercambio comercial e innovación (MIDEPLAN, 2014).

Si bien Costa Rica tiene gran potencial de innovación en Centroamérica, producto en otros elementos de los avances que a lo largo de varias décadas se han llevado a cabo en el sistema educativo, el uso de las tecnologías de la comunicación y la información, la capacidad de innovación y la tecnología disponible, debe atender con prontitud y de manera eficaz grandes retos en materia de infraestructura, fiscal, transporte, facilitación del comercio, entre otros, para mejorar la competitividad del país. 


\section{Referencias}

Álvarez, Isabel y Botella, Carlos (Eds.). (2012). Innovación y desarrollo: Retos para una sociedad global. Madrid: Fundación Carolina en coedición con Siglo XXI de España Editores.

Brunet, Ignasi y Baltar, Fabiola. (2010). Creación de empresas. Innovación e instituciones. España: RA-MA Editorial.

Consejo Nacional de Competitividad (2006) Agenda de competitividad de Costa Rica 2006-2016. Costa Rica. Recuperado de: http://spin-be.unesco.org.uy/ subida/documentosRelevantes/agenda(2).pdf

Diccionario LID Innovación. (2010). Ferran Amago y Borja Botanure (Edits, 1era Ed.). España: Lid EDITORIAL Empresarial.

Freeman, Christopher. (2008). Systems of innovation. Selected Essays in evolutionary economics. Publicación Cheltenham [etc.]: Edward Elgar.

Fundación COTEC para la Innovación Tecnológica. (2001). Gestión de la innovación y la tecnología en la empresa. Informes sobre el sistema español de innovación. Madrid: Autor.

Jaramillo Hernán, Lugones, Gustavo y Salazar, Mónica. (2001) MANUAL DEBOGOTÁ. Normalización de Indicadores de Innovación Tecnológica en América Latina y el Caribe Red Iberoamericana de Indicadores de Ciencia y Tecnología (RICYT) / Organización de Estados Americanos (OEA) / PROGRAMA CYTED COLCIENCIAS/OCYT. Recuperado de: file:///C:/Users/ RI/Documents/Downloads/bogota.pdf

León Balderrama, Jorge Inés. (2008). Determinantes de la participación de los investigadores en actividades de vinculación y transferencia de conocimiento. (Tesis de doctorado). Universidad Autónoma de Sinaloa, México.

Ministerio de Planificación Nacional y Política Económica. (2014). Plan Nacional de Desarrollo 2015-2018 “Alberto Cañas Escalante”. San José, Costa Rica: MIDEPLAN.

OECD. (2011). OECD Reviews of Regional Innovation. Catalonia, Spain. Paris: organisation for economic co-operation and development.

Organización de Cooperación y Desarrollo Económico/ Oficina de estadísticas de las comunidades europeas. (2005). Guía para la recogida e interpretación de datos sobre innovación. Manual de Oslo. Autor.

Porter, Michael E. (1991). Ventaja competitiva. Creación y sostenimiento de un desempeño superior. Argentina: Editorial Rei Argentina. 
Porter, Michael E. (2002). Estrategia competitiva. Técnicas para el análisis de los sectores industriales y de la competencia. México: Compañía editorial continental.

Porter, Michael E. (Enero-abril, 2005). ¿Qué es la competitividad? IESE Business School, Universidad de Navarra, l(1), Recuperado de: http://www.ieseinsight.com/doc.aspx?id=438\&ar=7\&idioma $=1$

The New Palgrave: A Dictionary of Economics (2008) Rachel Sangster, Publisher Ruth Lefèvre; Senior Editorial Coordinator. Recuperado de: http://www. dictionaryofeconomics.com/resources/about editors

World Economic Forum. (2014) The Global Competitiveness Report 2014-2015. Recuperado de: http://www3.weforum.org/docs/WEF_GlobalCompetitivenessReport_2014-15.pdf 